\title{
FREQUENCY AND ANTIBIOTIC RESISTANCE IN CLINICAL ISOLATES ACINETOBACTER BAUMANNII
}

\author{
D. Rukanova ${ }^{1^{*}}$, H. Djeneva ${ }^{1}$, K. Rachkova ${ }^{1,2}$, G. Lazarova ${ }^{1,2}$ \\ ${ }^{1}$ Department of Microbiology, Medical Faculty, Trakia University, Stara Zagora, Bulgaria \\ ¿'St. Kirkovich Hospital", Medical University, Stara Zagora, Bulgaria
}

\begin{abstract}
Multidrug resistant Gram-negative bacteria represent a global problem due to their global spread and growing distribution. They lead to major difficulties in therapy and to increased lethality and much more expensive treatment. Acinetobacter baumannii is a typical agent of this group of microorganisms.

The purpose of this study is to determine the frequency of distribution of A. baumannii in a variety of clinical materials of hospital patients and their antibiotic resistance to different groups of antibiotics used in medical practice.

Methods: A total of 371 strains A. baumannii were tested. They were isolated from clinical materials of patients of "St. Kirkovich Hospital"Medical University, Stara Zagora for a five-year period (2010-2014). The identification of the isolates was done by: routine methods, semi-automated Crystal system and automated API system. The disc diffusion method of Bauer Kirby was used to determine the antibiotic resistance.

Results: Among the 371 isolated strains of A. baumannii dominate those of surgical wounds $/ 40.2 \% /$, followed by isolates of the respiratory system (27.2\%). Antibiotic resistance to different antibiotic classes shows: Piperacillin - 92.3\% R, Ampicillin / Sulbactam - 69\% R, Ceftazidime - 91.6\% R, Cefepime - 91\% R, Imipenem - 32.7\% R, Meropenem - 48.9\% R, Amikacin - 76.2\% R, Ciprofloxacin -86.6\% R.
\end{abstract}

Key words: Acinetobacter baumannii, antimicrobial resistance

One of the main challenges of therapy of infections is the increasingly growing number of multidrug-resistant bacteria. Multiple antibiotic resistance occurs in both Grampositive and in Gram-negative bacteria. A significant problem are Gram-negative glucose-nonfermenting bacteria, to which the Acinetobacter baumannii belongs. The microorganism is characterized by a number of features that support its topicality: a high percentage of the isolates with multiple resistance to antimicrobials; the resistance is determined by several mechanisms simultaneously; tends to a constant increase of the resistance; permanent opening and quick spread of new mechanisms of resistance, incl. to carbapenems. This severely limits the therapeutic options $(1,2)$. A. baumannii is among the most common causes of serious infections - bacteraemia, pneumonia, soft tissue infections, urinary tract infections (3-7); in most cases these are hospital-acquired

\footnotetext{
*Correspondence to: D. Rukanova, Department of Microbiology, Medical faculty, Trakia University, 6000 Stara Zagora, Armeiska 11 Str. 042664 272; rukanova58@abv.bg
}

infections caused by isolates with the same types of resistance $(8,9)$; it requires constant monitoring, study of strains and summarizing the results in order to optimize antibiotic policy in hospitals.

The aim of this study is to determine the prevalence of $A$. baumannii in various clinical specimens from hospital patients, their antibiotic resistance to other classes of antibiotics used in medical practice and included in the Hospital antibiotic policy.

Materials and Methods: In the period 20102014 a total of 371 strains of $A$. baumannii were tested. The strains were isolated from materials of patients University Hospital "St. Kirkovich" Stara Zagora. Routine methods, semiautomated system Crystal (BBL) and automated API were used for the identification of the isolates. Disc method of Bauer Kirby was applied to determine the antibiotic resistance; interpretation of results was based on CLSI 2012.

Results and discussion: For the described 5year period a total of $371 \mathrm{~A}$. baumannii strains 
from different clinical materials, $(6.4 \%$ of the total clinically significant isolates) were analyzed. The study included only the first isolate of each patient.

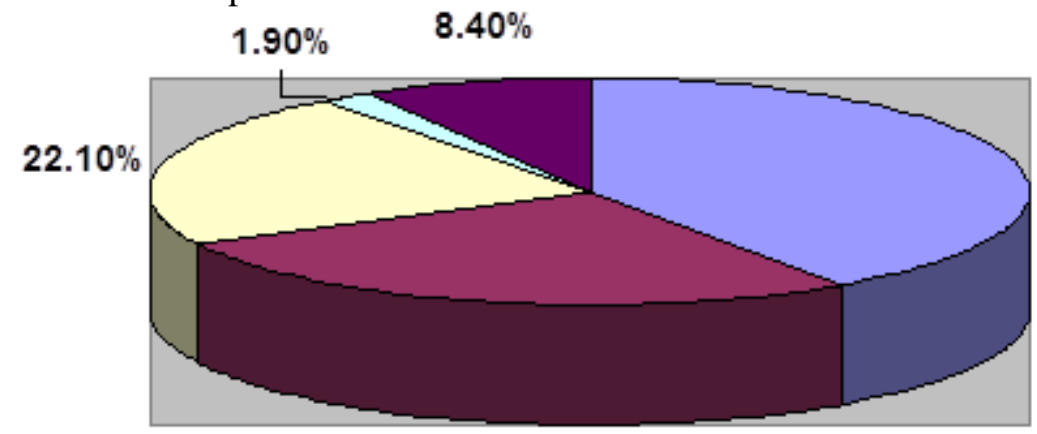

$27.20 \%$
The distribution of bacteria according to types of materials is described in Figure 1.

Figure 1. Distribution of A. baumannii in the types of examined materials

The results show that $40.2 \%$ of $A$. baumannii strains were isolated from surgical wounds, $27.2 \%$ - from the respiratory system and $22.1 \%$. - from urine cultures. A. baumannii is a typical nosocomial pathogen discovered both in materials from patients and the hospital environment objects (10). This explains the much higher isolation from surgical wounds versus other clinical specimens and especially wound isolates obtained from non-surgical clinics - 8.4\%. A. baumannii causes wound colonization and infection in patients with operative wounds, severe burns or trauma, especially associated with low hospital hygiene (11-13).

A. baumannii uroisolatites are more common in men $(70 \%)$; they are equally spread in both surgical and non-surgical patients. The high percentage of $A$. baumannii in patients from internal clinics is due to thear complex chronic urological history (patients who have undergone prior surgery or invasive procedures, mainly catheterization) (6).

In 7 patients $(1.9 \%)$ A. baumannii were isolated from blood culture. Those are patients in intensive care, where the microorganism is detected in other tested materials (tracheobronchial secretions or wound infection), excluded from the study. One strain A. baumannii was isolated from CSF. This is a Neurosurgery's patient with postoperative infection. There are references about cases of cerebrospinal shunt associated A.baumannii meningitis in neurosurgical patients (14).

The relative frequency of A.baumannii compared to other clinically significant microorganisms is shown in Figure 2.

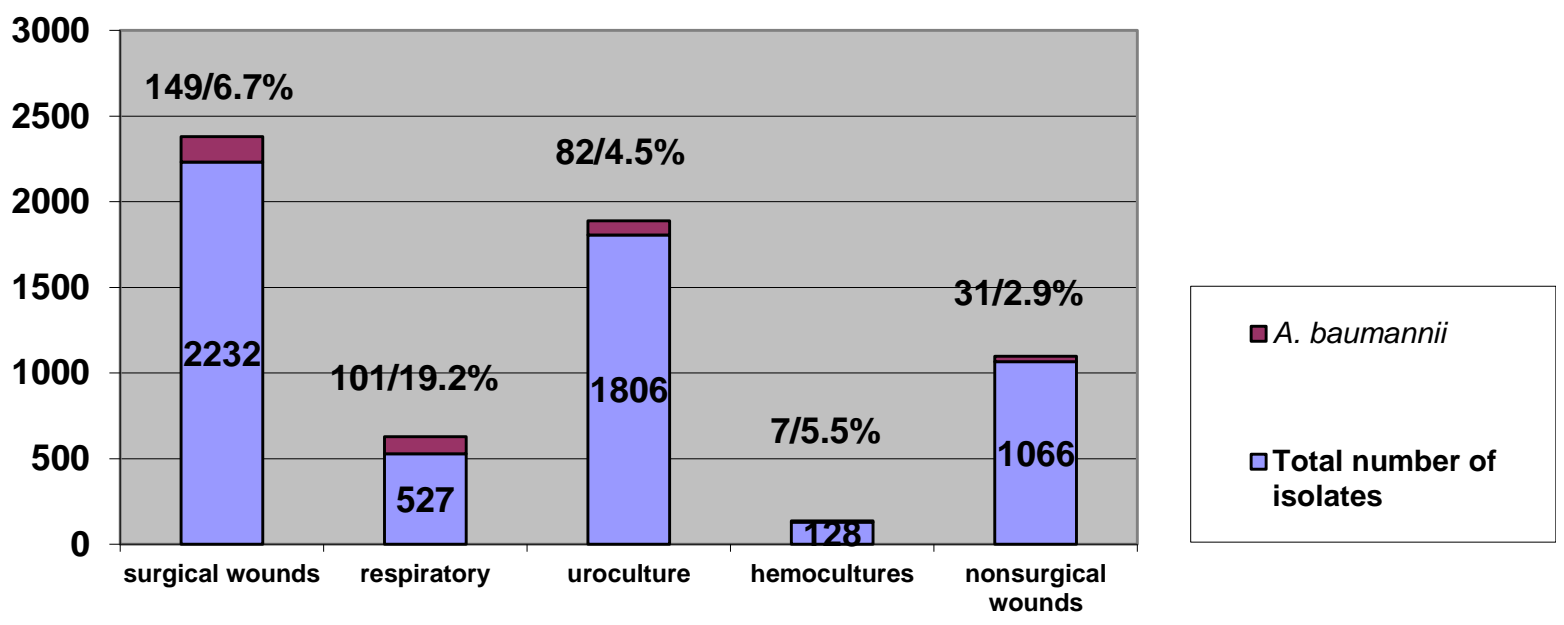

Figure 2. Proportion of $A$. baumannii against all clinically significant isolates by type materials

The frequency analysis of $A$. baumannii compared to other clinically significant microorganisms species from tested materials showed that $A$. baumannii was isolated most commonly from materials of the respiratory system $(9.2 \%)$. These are mainly isolates from the tracheo-bronchial secretions in patients in intensive care $(85.1 \%$ of cases). Ventilator associated pneumonia is the most common clinical manifestation of $A$. baumannii nosocomial infection (5-10\% in the US). Often nosocomial pneumonia is accompanied by 
bacteremia, which aggravates the prognosis (15).
The antibiotic resistance of the isolates is summarized below. The data is presented by type of clinical materials in Table 1.

Table 1. Antibiotic resistance of A. baumannii in \%

\begin{tabular}{|l|l|l|l|l|l|l|l|l|l|l|}
\hline $\begin{array}{l}\text { Antimicrobial agents } \\
\text { Clinical specimen }\end{array}$ & SAM & PIP & TZP & CAZ & FEP & MEM & IPM & AN & NN & CIP \\
\hline $\begin{array}{l}\text { Total number of } \\
\text { isolates }\end{array}$ & 69 & 92,3 & 81,5 & 91,6 & 91 & 48,9 & 32,7 & 76,2 & 13,6 & 86,6 \\
\hline Surgical wounds & 67 & 91.4 & 79.5 & 87.2 & 90.3 & 52.2 & 33.3 & 83.6 & 13 & 88.1 \\
\hline Respiratory system & 66.7 & 93.5 & 93.3 & 94.6 & 89.4 & 60 & 49.4 & 58.8 & 17.5 & 89.3 \\
\hline Urocultures & 71.6 & 89 & 75 & 92.1 & 92 & 29.2 & 7 & 81.3 & 12.5 & 85.7 \\
\hline Hemocultures & 20 & 100 & 100 & 100 & 100 & & 20 & 100 & & 100 \\
\hline $\begin{array}{l}\text { Nons-surgical } \\
\text { wounds }\end{array}$ & 80 & 88,5 & 71,4 & 92 & 84,2 & 31,3 & 22,2 & 73 & 0 & 72 \\
\hline
\end{tabular}

The high percentage of A. baumannii isolates show resistance (69-92.3\%) to the most frequently used antibiotics in practice penicillins, cephalosporins and fluoroquinolones. Such high values of resistance are not common in other Gramnegative bacteria, but are cited in other similar studies about A. baumannii (16), because it is typical for these species. (17-19). Multiple resistance of our A. baumannii isolates often make effective therapy difficult. A careful selection of antibiotic therapy is needed, if possible according to an antibiogram.

The resistance of $A$. baumannii to limited antibiotics, such as carbapenems, is relatively higher (MEM-48.9\%, IPM-32.7\%). In conccern with the carbapenems, our isolates have the same or lower resistance than other Bulgarian studies $(40 \%-82-85 \%)(4,16)$. The rapid appearance of strains of $A$. baumannii, resistant to all $\beta$-lactam antibiotics, including carbapenems, illustrates the potential of this organism to respond quickly to the selective pressure of the environment (17). Research through molecular genetic methods show even forming a Bulgarian branch of A. baumannii, isolated from various hospitals in the country, which produce different types of carbapenemases (8). Resistance to carbapenems shows that they can not be a good choice for empirical therapy or alternative therapy by multidrug resistant isolates. This requires continuous improvement of antibiotic policy in hospitals to limit the use of thirdgeneration cephalosporins and carbapenems as inducers of $\mathrm{ESBL}_{\mathrm{s}}$ and carbapenemases.

The resistance of A. baumannii is lowest the to Tobramycin - $13.6 \%$. In our hospital circulates a nosocomial strain, resistant to all other antibiotics and sensitive only to Tobramycin. The relatively low frequency of resistance to Tobramycin is reported in other similar studies too (16). Tobramycin is an opportunity for therapy and appropriate option for empirical therapy in patients at risk, especially in clinics with high isolation of A. baumannii.

Some of the isolates were tested to Colistin 56 strains $(15.6 \%)$, and to Tigecyclin - 97 strains $(27.1 \%)$. All these strains showed no resistance. Our results confirm the data of other authors for low $/ 1.3 \%$ for Colistin/ (16) to missing resistance of $A$. baumannii to these drugs. These antibiotics are a viable therapeutic alternative in multidrug resistant isolates. However, there are reports of in vitro increasing levels of resistance of $A$. baumannii to polymyxins and Tigecyclin (20-22).

Highest resistance showed isolates from the blood stream, followed by those of the respiratory system; the lowest is the resistance of the isolates from urine and wound infections.

Strains from blood cultures and respiratory system in all patients are an expression of hospital infections. The interpretation/generalization of the results shows that regardless of the type of research material, the treatment of $A$. baumannii isolates should be the same as the treatment of multidrug resistant microorganisms. In the development of therapeutic schemes hospitals should avoid selective pressure of 3generation cephalosporins and carbapenems, to slow down the increase of the resistance.

\section{CONCLUSION}

$>$ A. baumannii isolates showed a high frequency of resistance to penicillins, cephalosporins, fluoroquinolones and Amikacin, which requires an individual approach to therapy, after the result of the antibiograms. 
Desistance to carbapenems is high enough, which makes their use uncertain as empirical therapy.

$>$ The lower frequency of resistance to Tobramicin allows discussion as a treatment option and possibly empirical therapy.

$>$ Lack of resistance to Colistin u Tigecyclin of $A$. baumannii isolates suggests a safe alternative therapy.

A constant update of hospital antibiotic policy is needed to reduce the growth of antibiotic resistance.

\section{REFERENCES}

1. Bou, G., Cervero, G., Dominguez, M. A., Quereda, C. , and Martinez-Beltran, J, Characterization of a nosocomial outbreak caused by a multiresistant Acinetobacter baumannii strain with a carbapenemhydrolyzing enzyme: high-level carbapenem resistance in A. baumannii is not due solely to the presence of betalactamases. J. Clin. Microbiol., 38:32993305, 2000.

2. Perilli, M., Felici, A., Oratore, A., Cornaglia, G., Bonfiglio, G., Rossolini, G. M., Amicosante. G., Characterization of the chromosomal cephalosporinases produced by Acinetobacter lwoffii and Acinetobacter baumannii clinical isolates. Antimicrob. Agents Chemother. ,40:715-719,1996.

3. Lazarova, G., Rachkova, K., Rukanova, D., Boicheva, M., Yordanova, R., Djeneva, H., Teneva, M., Etiological and antimicrobial resistance of isolates from surgical infections among patients in hospital "Prof. dr. St. Kirkovich" University-Stara Zagora for 2013. XII Congress of Bulgarian Association of Microbiologists, 2014, Abstract Book, p.19.

4. Keuleyan, E., Tete, Sh., Valentinova, M., Baycheva, M., Panayotova, D., Problematic antibiotic resistant microorganisms and consumption of antibiotics in a multiprofile hospital. XII Congress of Bulgarian Association of Microbiologists, 2014, Abstract Book, p.15.

5. Peleg, A.Y., Seifert, H., Paterson, D. L., Acinetobacter baumannii:emergence of a successful pathogen. Clin. Microbiol. Rev., 21,538-582,2008.

6. Dijkshoorn, L., Nemec, A., and Seifert, H., An increasing threatin hospitals: multidrugresistant Acinetobacter baumannii. Nat. Rev.Microbiol., 5, 939-951, 2007.

7. Garcı'a-Garmendia, J. L., Ortiz-Leyba, C., Garnacho-Montero, J., Jime'-nez-Jime'nez, F. J., Pe'rez-Paredes, C., et al., Risk factors for Acinetobacter baumannii nosocomial bacteremia in critically ill patients: a cohort study. Clin. Infect. Dis., 33, 939-946.-13, 2001

8. Vacheva-Dobrevska, R., Ivanov, I., Higgins, P. et all, Development of resistance, molecular characterisation and clonal spread of carbapenem resistant $A$. baumannii in Bulgarian hospitals. XII Congress of Bulgarian Association of Microbiologists, 2014, Abstract Book, p.72.

9. Teocharov, P., Vladimirova, N., Dobreva, E. et all, The national reference centre for healthcare-associated infections (NRCHAI). Activities in 2014. XIII Congress of Bulgarian Association of Microbiologists, 2015, Abstract Book, p.14.

10.Stamatova, I., Petrova, A., Murdjeva, M., Microbiological analysis of species from hospital "St. George" -Plovdiv-a strategy to prevent and control hospital acquired infections. XII Congress of Bulgarian Association of Microbiologists, 2014, Abstract Book, p.46.

11.Scott, P., Deye, G., Srinivasan, A., Murray, C., Moran, K., et al., An outbreak of multidrug-resistant Acinetobacter baumannii-calcoaceticuscomplex infection in the US military health care system associated with military operations in Iraq. Clin. Infect. Dis., 44, 1577-1584, 2007

12.Sebeny, P. J., Riddle, M. S., and Petersen, $\mathrm{K}$., Acinetobacter baumannii skin and softtissue infection associated with war trauma. Clin.Infect. Dis., 47, 444-449, 2008

13.Wisplinghoff, H., Bischoff, T., Tallent, S. M., Seifert, H., Wenzel, R.P., et al. , Nosocomial bloodstream infections in US hospitals: analysis of 24,179 cases from a prospective nationwide surveillance study.Clin. Infect. Dis., 39, 309-317, 2004.

14.Kim, B. N., Peleg, A. Y., Lodise, T. P., Lipman, J., Li, J., et al. , Management of meningitis due to antibiotic-resistant Acinetobacter species.Lancet Infect. Dis. ,9, 245-255, 2009.

15.Seifert, H., Strate, A., and Pulverer, G. Nosocomial bacteremia due to Acinetobacter baumannii. Clinical features, epidemiology, andpredictors of mortality. Medicine (Baltimore), 74, 340-349, 1995

16.Savov, E., Keuleyan, E., Borisova, N., Gergova, I., Ronkova, G., Trifonova, A., In vitro study of the resistance of problematic for hospital infectious pathology microorganisms to antimicrobial drugs. Trakia Journal of Sciences, Vol. 8. No 2, 2010.

17.Bacher, J. M., D. Metzgar, D., de CrecyLagard, V., Rapid evolution of diminished transformability in Acinetobacter baylyi. J. Bacteriol., 188:8534-8542, 2006. 
18.Perez, F.,. Hujer, A. M., Hujer, K. M,. Decker, B. K., Rather, P. N., Bonomo, R. A.,Global challenge of multidrug-resistant Acinetobacter baumannii. Antimicrob. Agents Chemother.,51:3471-3484, 2007.

19.Poirel, L., Nordmann, P., Carbapenem resistance in Acinetobacter baumannii: mechanisms and epidemiology. Clin. Microbiol. Infect., 12:826-836, 2006.

20.Gales, A. C., Jones, R. N., Sader, H. S., Global assessment of the antimicrobial activity of polymyxin B against 54,731 clinical isolates of gram-negative bacilli: report from the SENTRY antimicrobial surveillance programme (2001-2004), Clin. Microbiol. Infect. 12:315-321, 2004.

21.Li, J., Rayner, C. R, Nation, R. L., Owen, R. J., Spelman, D., Tan, K. E., Liolios, L., Heteroresistance to colistin in multidrugresistant Acinetobacter baumannii. Antimicrob. Agents Chemother., 50:29462950, 2006.

22.Peleg, A. Y., Adams, J. , Paterson, D. L., Tigecycline efflux as a mechanism for nonsusceptibility in Acinetobacter baumannii. Antimicrob. Agents Chemother., 51:2065-2069, 2007. 